\title{
504.
}

\section{ON THE MECHANICAL DESCRIPTION OF CERTAIN SEXTIC CURVES.}

[From the Proceedings of the London Mathematical Society, vol. Iv. (1871-1873), pp. 105-111. Read April 11, 1872.]

THE curves in question might be taken to be those described by a point $C$ rigidly connected with points $A$ and $B$, each of which describes a circle: but the construction is considered under a somewhat more general form. I consider a quadrilateral, the sides of which are $a, b, c, d$, and the inclinations of these to a fixed line $\alpha, \beta, \gamma, \delta$. This being so, if $a, b, c, d$, and one of the angles, say $\delta$, are constant, then we have between the three variable angles the relations

$$
\begin{aligned}
& a \cos \alpha+b \cos \beta+c \cos \gamma+d \cos \delta=0, \\
& a \sin \alpha+b \sin \beta+c \sin \gamma+d \sin \delta=0,
\end{aligned}
$$

giving rise to a single relation between any two of the variable angles; and we consider a curve such that the coordinates $x, y$ of any point thereof are given linear functions of the sines and cosines of the three variable angles, or, what is the same thing, of the sines and cosines of any two of these angles. We thus unite together what would otherwise be distinct cases; for everything is symmetrical in regard to the sides $a, b, c$ and the corresponding variable angles $\alpha, \beta, \gamma$, irrespectively of the order of succession of these sides: and we can thus, in the discussion of the curve, employ any two at pleasure, say $\alpha, \beta$, of the variable angles, without determining whether the sides $a, b$ are contiguous or opposite.

Eliminating, then, the variable angle $\gamma$, we obtain between $\alpha, \beta$ a relation which, if we write therein $\tan \frac{1}{2} \alpha=u, \tan \frac{1}{2} \beta=v$, takes the form $(* \gamma u, 1)^{2}(v, 1)^{2}=0$; viz. either of the variables $u, v$ is expressible rationally in terms of the other of them and of the root of a quartic function thereof; say $v$ is a rational function of $u$ and $\sqrt{ } U$. And 
hence a curve for which the coordinates $x, y$ are rational functions of $u, v$, is a curve having a deficiency $D=1$, or, what is the same thing, having a number of dps. less by unity than the maximum number $\left\{=\frac{1}{2}(n-1)(n-2)\right.$, if $n$ be the order of the curve $\}$.

It will further appear that the relation $\left(*(u, 1)^{2} .(v, 1)^{2}=0\right.$ is satisfied by the values $u=v=i$ and $u=v=-i$ (if, as usual, $i=\sqrt{-1}$ ).

In the curve in question, the coordinates $(x, y)$ are given linear functions of the sines and cosines of $\alpha, \beta$; and if we make the curve meet an arbitrary line $A x+B y+C=0$, we obtain between the sines and cosines of $\alpha, \beta$ a linear relation which, substituting therein the expressions in terms of $u, v$, takes the form

$$
(* \gamma u, 1)^{2} \cdot\left(1+v^{2}\right)+(* \gamma v, 1)^{2} \cdot\left(1+u^{2}\right)=0,
$$

viz. this is a relation of the form $(+\gamma u, 1)^{2}(v, 1)^{2}=0$, such that it is satisfied by the four sets of values $u= \pm i, v= \pm i$, and therefore in particular by the values $u=v=i$ and $u=v=-i$.

Hence, considering the intersections of the curve by the arbitrary line, the values of $(u, v)$ are given by the two equations $(* \gamma u, 1)^{2}(v, 1)^{2}=0,(+\gamma u, 1)^{2}(v, 1)^{2}=0$; - these, regarding for a moment $u, v$ as ordinary rectangular coordinates, represent each of them a quartic curve. having two dps. at infinity on the axes $u=0, v=0$ respectively: each of these points reckons therefore as 4 intersections, and the number of the remaining intersections therefore is $4.4-2.4,=8$. But, by what precedes, the two quartic curves have also in common the points $u=v=i$ and $u=v=-i$; and rejecting these, there remain $8-2,=6$ intersections.

The conclusion is, that the curve is a sextic curve of deficiency 1 , that is, having 9 dps. The reasoning may be presented under a slightly different form as follows: regarding $u, v$ as coordinates, we have the curve $(* \chi u, 1)^{2}(v, 1)^{2}=0$, a binodal quartic curve, and having therefore the deficiency 1 ; the curve passes, as above-mentioned, through the points $u=v=i$ and $u=v=-i$. The required curve is obtained as a transformation of the quartic curve by formulæ of the form $x: y: z(=1)=P: Q: R$, where $P, Q$, and $R\left\{=\left(1+u^{2}\right)\left(1+v^{2}\right)\right\}$ are quartic functions of the coordinates $u, v$, such that $P=0, Q=0, R=0$ are each of them a quartic curve passing twice through each of the nodes and once through each of the before-mentioned points, $(u=v=i)$ and $(u=v=-i)$, of the binodal quartic curve. Hence the curve in question is a curve of the order $4.4-2.4-2.1,=6$, and having the same deficiency as the binodal quartic, that is, the deficiency is $=1$.

I observe that the sextic curve does not, in general, pass through the circular points at infinity, but it intersects the line at infinity in three distinct pairs of points; one of these, or all three of them, (but not two pairs only,) may coincide with the circular points at infinity, the circular points at infinity being, in the latter case, triple points, or the curve being tricircular: this will appear presently.

To obtain the foregoing equation $(* \gamma u, 1)^{2}(v, 1)^{2}=0$, the elimination of $\gamma$ gives $(a \cos \alpha+b \cos \beta+d \cos \delta)^{2}+(a \sin \alpha+b \sin \beta+d \sin \delta)^{2}=c^{2}$, 
that is

$$
a^{2}+b^{2}-c^{2}+d^{2}+2 a b \cos (\alpha-\beta)+2 a d \cos (\alpha-\delta)+2 b d \cos (\beta-\delta)=0 ;
$$

or, substituting herein the values

$$
\cos \alpha=\frac{1-u^{2}}{1+u^{2}}, \quad \sin \alpha=\frac{2 u}{1+u^{2}}, \quad \cos \beta=\frac{1-v^{2}}{1+v^{2}}, \quad \sin \beta=\frac{2 v}{1+v^{2}},
$$

\begin{tabular}{|c|c|c|c|}
\hline & $(a+b)^{2}-2(a+b) d \cos \delta+d^{2}-c^{2}$ & $2 a d \sin \delta$ & $(a-b)^{2}+2(a-b) d \cos \delta+d^{2}-c^{2}$ \\
\hline & $2 b d \sin \delta$ & $2 a b$ & $2 b d \sin \delta$ \\
\hline$v^{2}$ & $(a-b)^{2}-2(a-b) d \cos \delta+d^{2}-c^{2}$ & $2 a d \sin \delta$ & $(a+b)^{2}+2(a+b) d \cos \delta+d^{2}-c^{2}$ \\
\hline
\end{tabular}

this is easily found to be $0=$

or writing, for greater convenience, $\delta=0$, that is, taking $\alpha, \beta, \gamma$ to be the inclinations of the sides $a, b, c$ to the fixed side $d$, this is $0=$

\begin{tabular}{|c|c|c|}
\hline 1 & 1 & \multicolumn{2}{c}{$2 u$} & $u^{2}$ \\
\hline $2 v$ & 0 & $(a-b+d)^{2}-c^{2}$ \\
\hline & 0 & 0 \\
\hline & $2 a b$ & \\
\hline$(a-b-d)^{2}-c^{2}$ & 0 & $(a+b+d)^{2}-c^{2}$ \\
\hline
\end{tabular}

or say this is

$$
\left(A+B u^{2}\right)+8 a b u v+v^{2}\left(C+D u^{2}\right)=0,
$$

where, writing for shortness

then

$$
\begin{aligned}
& a+d-b-c=\lambda, \quad-a+b+c+d=\lambda^{\prime} \\
& b+d-c-a=\mu,-b+c+d+a=\mu^{\prime} \\
& c+d-a-b=\nu,-c+d+a+b=\nu^{\prime} \\
& a+b+c+d=\sigma, \quad-d+a+b+c=\rho^{\prime}
\end{aligned}
$$

$$
A=-\nu \rho^{\prime}, \quad B=\lambda \mu^{\prime}, \quad C=\lambda^{\prime} \mu, \quad D=\nu^{\prime} \sigma .
$$

We at once verify that the equation is satisfied by $u=v= \pm i$, viz. this will be so if only

$$
A-B-C+D-8 a b=0
$$


and we have

$$
\begin{aligned}
& A+D=2(a+b)^{2}+2 d^{2}-2 c^{2} \\
& B+C=2(a-b)^{2}+2 d^{2}-2 c^{2}
\end{aligned}
$$

whence the relation in question.

Writing $u=i$, we have

whence

$$
\begin{aligned}
\{v(C-D)+4 a b i\}^{2} & =-16 a^{2} b^{2}-(A-B)(C-D) \\
& =-\frac{1}{4}(A-B+C-D)^{2} \\
v(C-D) & = \pm \frac{1}{2} i\{\mp 8 a b+A-B+C-D\} \\
& =i(C-D) \text { or }-i(A-B)
\end{aligned}
$$

so that, corresponding to $u=i$, we have the values $v=i$ and $v=-i \frac{A-B}{C-D}$; and similarly, corresponding to $u=-i$, the values $v=-i$ and $v=i \frac{A-B}{C-D}$.

And in like manner, corresponding to $v=i$, we have the values $u=i$ and $u=-i \frac{A-C}{B-D}$; and to $v=-i$, the values $u=-i$ and $u=i \frac{A-C}{B-D}$.

It is easy to show that, if $u=i+\epsilon, v=i+\zeta$ are the values consecutive to $u=v=i$, then $a \epsilon+b \zeta=0$ : in fact, substituting the foregoing values in the relation between $u, v$, and writing for $8 a b$ its value, $=A-B-C+D$, we have

$$
A+B(-1+2 i \epsilon)+(A-B-C+D)\{-1+i(\epsilon+\zeta)\}+C(-1+2 i \zeta)+D\{1-2 i(\epsilon+\zeta)\}=0
$$

which is

$$
=\epsilon(A+B-C-D)+\zeta(A-B+C-D)=0 ;
$$

or finally $a \epsilon+b \xi=0$. And similarly, if $u=-i+\epsilon, v=-i+\zeta$ are the values consecutive to $u=v=-i$, then we have the same relation $a \epsilon+b \zeta=0$.

The points at infinity on the sextic curve are those for which $1+u^{2}$ or $1+v^{2}$, or each of these, is $=0$; viz. the values of $u, v$ for the six points are

$$
\begin{array}{llll}
u=i+\epsilon, & -i-\epsilon, \quad i \quad, \quad-i \quad-i\left(\frac{A-C}{B-D}\right), & i\left(\frac{A-C}{B-D}\right) ; \\
v=i+\zeta, & -i-\zeta, & -i \frac{A-B}{C-D}, \quad i \frac{A-B}{C-D}, \quad i \quad,-i,
\end{array}
$$

where, instead of $u=v=i$ and $u=v=-i$, I have written down the consecutive values of $u, v$, and as before $\epsilon, \zeta$ are infinitesimals such that $a \epsilon+b \zeta=0$.

Suppose that the coordinates $x, y$ of a point on the sextic curve are

$$
\begin{aligned}
& x=a \cos \alpha+c \sin \alpha+b \cos \beta+d \sin \beta, \\
& y=a^{\prime} \cos \alpha+c^{\prime} \sin \alpha+b^{\prime} \cos \beta+d^{\prime} \sin \beta
\end{aligned}
$$


then, if $u= \pm(i+\epsilon), \cos \alpha=\frac{-i}{\epsilon}, \sin \alpha=\frac{ \pm 1}{\epsilon}$, and similarly if $v= \pm(i+\zeta)$, then $\cos \beta=-\frac{i}{\zeta}$, $\sin \beta=\frac{ \pm 1}{\zeta}$. Hence the points at infinity of the sextic curve are as follows:

$$
\text { 1.. } \begin{aligned}
u & = \pm(i+\epsilon), \quad v \text { not }= \pm(i+\zeta) \\
x & =\frac{-a i \pm c}{\epsilon}, \quad y=\frac{-\mathrm{a}^{\prime} i \pm \mathrm{c}^{\prime}}{\epsilon}, \text { first pair of points }
\end{aligned}
$$

$2^{\circ} . \quad v= \pm(i+\zeta), \quad u$ not $= \pm(i+\epsilon)$,

$x=\frac{-\mathrm{b} i \pm \mathrm{d}}{\zeta}, y=\frac{-\mathrm{b}^{\prime} i \pm \mathrm{d}^{\prime}}{\zeta}$, second pair of points ;

3. $u= \pm(i+\epsilon), \quad v= \pm(i+\zeta)$,

$$
x=\frac{-\mathrm{a} i \pm \mathrm{c}}{\epsilon}+\frac{-\mathrm{bi \pm d}}{\zeta}, y=-\frac{\mathrm{a}^{\prime} i \pm \mathrm{c}^{\prime}}{\epsilon}+\frac{-\mathrm{b}^{\prime} i \pm \mathrm{d}}{\zeta}
$$

$$
a \epsilon+b \zeta=0 \text {, as above, third pair of points ; }
$$

which six points are in general distinct from each other, and from the circular points at infinity.

The foregoing values of $x, y$ may be said to be "circular quoad $\alpha$," if $a=c^{\prime}$, $a^{\prime}=-c$; and similarly to be "circular quoad $\beta$," if $b=d^{\prime}, b^{\prime}=-d$.

And we see at once that if the values are circular quoad $\alpha$, then the first pair of points coincide with the circular points at infinity; and that, in like manner, if the values are circular quoad $\beta$, then the second pair of points coincide with the circular points at infinity; but if the values are circular quoad $\alpha$ and $\beta$ respectively, then each of the three pairs of points coincides with the circular points at infinity: so that these are then triple points on the curve; or the curve is tricircular, having besides the two triple points, 3 dps.

The relation between $u, v$ gives

$$
\left\{v\left(C+D u^{2}\right)+4 a b u\right\}^{2}=16 a^{2} b^{2} \cdot u^{2}-\left(A+B u^{2}\right)\left(C+D u^{2}\right),
$$

and it thus appears that if any one of the functions $A, B, C, D$ is $=0$, the function under the radical sign is a mere linear function of $u^{2}$, say it is $L+M u^{2}$; introducing a new parameter $\theta$ such that $u=\sqrt{ }\left(\frac{L}{M}\right) \frac{2 \theta}{1+\theta^{2}}$, we have $\sqrt{L+M u^{2}}=\sqrt{L} \frac{1+\theta^{2}}{1-\theta^{2}}$, and consequently $u, v$ are each of them a rational function of $\theta$. Hence, when any one of the relations in question is satisfied, or say, when $a+d=b+c, b+d=c+a$, or $c+d=a+b$, the curve becomes unicursal: there is no diminution of the order, and the curve is consequently a unicursal sextic, or sextic with $10 \mathrm{dps}$. 
It would at first sight appear that the curve might become unicursal in a different manner; viz. it would be unicursal if

$$
16 a^{2} b^{2} u^{2}-\left(A+B u^{2}\right)\left(C+D u^{2}\right)
$$

was a perfect square; but this is only the case when one of the four sides $a, b, c, d$ is $=0$. The condition in fact is

that is

$$
A D+B C-16 a^{2} b^{2}=2 \sqrt{A B C D}
$$

$$
\lambda \lambda^{\prime} \mu \mu^{\prime}-\nu \nu^{\prime} \rho^{\prime} \sigma-16 a^{2} b^{2}=2 \sqrt{-\lambda \lambda^{\prime} \mu \mu^{\prime} \rho^{\prime} \sigma} ;
$$

where, putting for shortness $M=d^{2}-a^{2}-b^{2}-c^{2}$, we have

$$
\begin{array}{r}
\lambda \lambda^{\prime}=M-2 b c+2 c a+2 a b, \\
\mu \mu^{\prime}=M+2 b c-2 c a+2 a b, \\
\nu \nu^{\prime}=M+2 b c+2 c a-2 a b, \\
-\rho^{\prime} \sigma=M-2 b c-2 c a-2 a b ;
\end{array}
$$

and thence

$$
\begin{aligned}
\lambda \lambda^{\prime} \mu \mu^{\prime} & =M^{2}-4 b^{2} c^{2}-4 c^{2} a^{2}+4 a^{2} b^{2}+4 a b M+8 c^{2} a b, \\
-\nu \nu^{\prime} \rho^{\prime} \sigma & =M^{2}-4 b^{2} c^{2}-4 c^{2} a^{2}+4 a^{2} b^{2}-4 a b M-8 c^{2} a b,
\end{aligned}
$$

and the equation thus becomes

$$
M^{2}-4 b^{2} c^{2}-4 c^{2} a^{2}-4 a^{2} b^{2}=\sqrt{\left(M^{2}-4 b^{2} c^{2}-4 c^{2} a^{2}+4 a^{2} b^{2}\right)^{2}-16 a^{2} b^{2}\left(M+2 c^{2}\right)^{2}},
$$

viz. putting for a moment $X=M^{2}-4 c^{2}\left(a^{2}+b^{2}\right)$, this is

that is

$$
\left(X-4 a^{2} b^{2}\right)^{2}=\left(X+4 a^{2} b^{2}\right)^{2}-16 a^{2} b^{2}\left(M+2 c^{2}\right)^{2},
$$

$$
16 a^{2} b^{2}\left\{X-\left(M+2 c^{2}\right)^{2}\right\}=0 ;
$$

or, substituting for $X$ its value, the equation is

$$
64 a^{2} b^{2} c^{2}\left(M+a^{2}+b^{2}+c^{2}\right)=0,
$$

that is $a^{2} b^{2} c^{2} d^{2}=0$.

We may have simultaneously $1^{\circ}, a=d, b=c ; 2^{\circ}, b=d, a=c ; 3^{\circ}, c=d, a=b$; the three cases are really equivalent, but the results present themselves in different forms.

$1^{\circ}$. Here $A=0, B=4 a(a-b), C=0, D=4 a(a+b)$; the relation between $u, v$ contains the factor $u$, and throwing this out, and also the constant factor $4 a$, it is

$$
u\left[(a-b)+(a+b) v^{2}\right]+2 b v=0,
$$

viz. $u$ is given as a rational function of $v$. 
$2^{\circ}$. Here $A=0, B=0, C=4 b(b-a), D=4 b(b+a)$; the equation contains the factor $v$, and throwing out this and also the constant factor $4 b$, the equation is

$$
v\left[(b-a)+(b+a) u^{2}\right]+2 a u=0,
$$

viz. $v$ is given as a rational function of $u$.

$3^{\circ}$. Here $A=4 a(a-c), B=0, C=0, D=4 a(a+c)$; or, dividing by $4 a$, the equation is

$$
(a-c)+2 a u v+(a+c) u^{2} v^{2}=0
$$

viz. this is

$$
(u v+1)[(a+c) u v+a-c]=0,
$$

which may be reduced to

$$
(a+c) u v+a-c=0,
$$

giving $u$ or $v$ each a rational function of the other.

I do not discuss the theory in detail, but only remark that in each case there is a conic thrown off, and that in place of the sextic we have a unicursal (or trinodal) quartic curve. 